\title{
Factors Associated With Late Antenatal Enrolment Among Pregnant Women Aged 15-49 Years At Masindi Hospital
}

\author{
Kizito Omona $[\mathrm{PhD}]^{1 *}$; Immaculate Kemigisha ${ }^{1}$; Tymothy Mugume ${ }^{1}$; \\ Asaph Muhanguzi ${ }^{1}$; Sarah Lubega ${ }^{1}$; Obed Atuhaire ${ }^{1}$ \\ ${ }^{1}$ Faculty of Health Sciences, Uganda Martyrs University, Uganda \\ Corresponding Author: Dr Omona Kizito, Lecturer. E-mail: komona@umu.ac.ug, Tel: +256706464873 \\ DOI: 10.47760/cognizance.2021.v01i03.003
}

\begin{abstract}
Introduction: Early ANC attendance is vital for early identification of pregnancy related health risks to either mother or baby or both, and interventions instituted. This results in good delivery outcomes.
\end{abstract}

Objective: The main objective of this study was to assess the factors associated with late antenatal care enrollment among pregnant mothers of 15-49 years at Masindi district hospital.

Methodology: Analytical cross-sectional study using quantitative method was used. The study included 380participants. A semi-structured questionnaire was used and SPSS 16.0software was used to analyses the results.

Results: The proportion of late enrollment into ANC was $81.1 \%$ since they made their first ANC visit after 12 weeks. Late enrolment for ANC was independently associated with being Protestants (AOR-9.043; 95\% CI-1.089-90.821; p-0.042), knowledge that the right time for first ANC is after 28 weeks of pregnancy (AOR-3.019; 95\% CI-1.001-6.342; p-0.000), knowledge that right time to seek first pregnancy was immediately (AOR-0.007; 95\% CI.001-.093; p-0.007), knowledge on the recommended number of ANC visits as four times(AOR-0.777; 95\% CI-294-.890; p-0.001), among others. It was also associated with disagreeing that Antenatal care follow-up is good to monitor mother's and fetus's health (AOR-33.189; 95\% CI-2.639-42.419; p-0.019), strongly disagreeing that Antenatal care follow-up is good to monitor mother's and fetus's health (AOR-36.348; 95\% CI-4.36592.711; $p-0.009)$.

Conclusion: The general proportion of late enrollment for ANC was high at $81.1 \%$ and knowledge of women on ANC, social demographic characterizes attitude and played key role in women attending ANC on time.

Keywords: Late ANC Visit, Pregnant women, Sulphadoxinepyremethamine, Attitude, Malaria

\section{INTRODUCTION}

1.1 Background of the study

Antenatal care can be defined as the care provided by skilled health-care professionals to pregnant women and adolescent girls in order to ensure the best health conditions for both mother and baby during pregnancy(1). The same author denotes that within the continuum of 


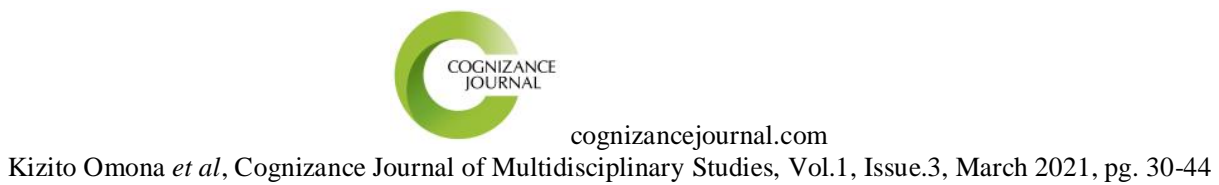

reproductive health care, antenatal care (ANC) provides a platform for important health-care functions, including health promotion, screening and diagnosis, and disease prevention. Pregnancy is an important phase in the life of a woman, family and society. During this period, antenatal care plays a crucial role for both mother and her unborn child to ensure good health (2). As one of the four pillars of the Safe Motherhood Initiative, when started early, it is key in improving maternal and new born health hence contributing to the SDG target 3.1 which is: to reduce the global maternal mortality ratio to less than 70 per 100000 live births by 2030 (3).

Women who attend ANC to have 'positive pregnancy experience which can create the foundations for healthy motherhood'(1). A study (4) revealed that key elements for a positive pregnancy experience include: maintaining physical and socio-cultural normality; maintaining a healthy pregnancy for mother and baby (preventing and treating risks, illness and death); having an effective transition to positive labor and birth; and achieving positive motherhood (maternal self-esteem, competence and autonomy). It (5) further noted that that ANC reduces maternal and perinatal morbidity and mortality both directly by detection and treatment of pregnancy-related complications, and indirectly, through the identification of women and girls at increased risk of developing complications during labor and delivery, hence ensuring referral to an appropriate level of care. Some of the recommendations by (6) emphasize that antenatal care should be started as soon as the mother has missed one menstrual period followed by eight ANC visits which are spread out over her pregnancy period. This should be done by a skilled health worker at a health facility with the aim of reducing perinatal mortality and improving the mother's experience of care. The Antenatal care visits are packaged in a way that vital actions are carried out on the mother.

It has been estimated that 25 percent of maternal deaths occur during pregnancy, with variability between countries depending on the prevalence of unsafe abortion, violence, and disease in the area (7). However, adequate antenatal care attendance has shown to reduce preventable causes of death and disability for both the pregnant mother and unborn child (8). This is achievable through treatment of health related conditions such as eclampsia, malaria, anemia, HIV and perinatal per vaginal bleeding; and referrals where necessary (9). The same author further stated that $94 \%$ occurred in low-resource settings like Uganda with subSaharan Africa contributing about $86 \%$. The five outstanding causes of these deaths were: severe bleeding, infections, high blood pressure during pregnancy (pre-eclampsia and eclampsia), complications from delivery and unsafe abortion (3).

In Low income countries like Uganda, ANC attendance is still very low as showed by some reports from (11). The 2016 UBOS report mentions that $95 \%$ of pregnant women got at least one antenatal visit; $60 \%$ received four and more visit with Bunyoro having the at least four or more ANC attendance at $45 \%$ which indicate that Bunyoro has the lowest percentage. Most women in Uganda have registered late ANC attendance, averagely at 5.5 months of pregnancy and do not complete the required four visits (12). Late booking to antenatal care is associated with poor outcomes for both mothers and babies(13).

Nevertheless, a large number of mothers in Uganda enroll late for first antenatal visit; contrary to the WHO recommendations(14). This leads to mothers missing out on antennal packages suggested for the first months and they are unable to finish the recommended eight antenatal visits(7). When the full antenatal package is missed, some of these mothers may develop complications before and during labor; leading to disability or death of the mother or baby or both; that would have be identified and treated earlier if the mother attended 


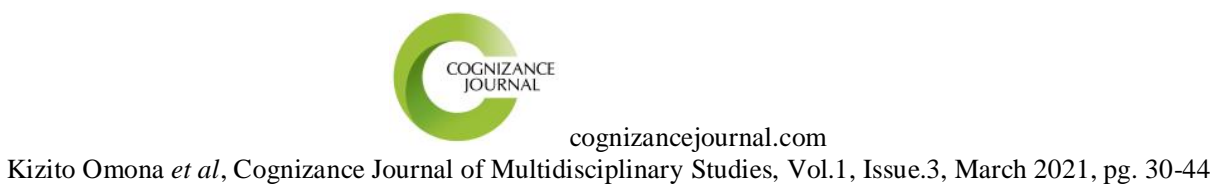

antenatal. This causes grief to the family, community and country at large due to reduced productivity and large expenses on such mothers (3).

The Uganda government has adopted and implemented WHO guidelines on ANC, recruited health workers to deliver ANC services and providing funding to these services. Some local NGOs and private health facilities in Uganda also provide ANC. With all this effort, many mothers still enrol for ANC late in the months as in the case of our study area the Bunyoro region. Therefore, this study intends to assess the factors leading late ANC enrolment among mothers attending ANC clinic at Masindi Hospital.

\subsection{Study area}

The study was carried out at Masindi Hospital. The hospital is located in the town of Masindi, in Masindi District, in Bunyoro sub-region, about 66 kilometres (41 mi), by road, northeast of Hoima Regional Referral Hospital. Built in 1922 as a health aid post of the workers of the East African Railways, by 1935 it was acquired by the colonial government and developed into a Health Center IV facility. In 1965 it was handed over to the government of Uganda and in 1988, it was upgraded to a 100-bed general hospital. 54 gynecology beds were added in 2008, to bring the bed capacity to 154. Patients served come from the districts of Masindi, Buliisa, Nakasongola, Nakaseke, Hoima and Nebbi.

\subsection{Broad objective}

To assess the factors associated with late antenatal enrollment among pregnant mothers aged 15-49 years at Masindi hospital

\subsection{Specific objectives}

(1) To determine the time of enrolment for Antenatal Care (ANC) among pregnant women aged 15-49 Years at Masindi Hospital

(2) To determine the socio-demographic factors associated with late ANC enrolment among pregnant mothers aged 15-49 years in Masindi Hospital

(3) To establish whether knowledge of Mothers on antenatal care services was associated with late ANC visit in Masindi Hospital

(4) To establish whether attitudes of mothers towards ANC services was associated with late ANC visit in Masindi Hospital

\section{METHODOLOGY}

\subsection{Study design}

This was analytical cross-sectional study employing quantitative methods of data collections.

\subsection{Study population}

This study focused on expectant mothers aged 15 to 49 years attending antenatal at Masindi Hospital.

\subsection{Inclusion and Exclusion Criteria}

\subsubsection{Inclusion criteria}

Pregnant women 15 to 49 years of age, who have had their first antenatal visit after 14 weeks of gestation. This includes women who have come for follow-up visits, though their first visit was after 14 weeks. This is because the first trimester pregnancy stage is the fastest 


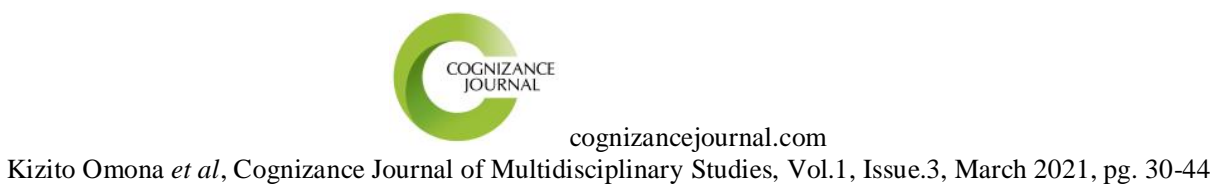

developmental period of the fetus, in which all organs become well developed and needs special attention.

\subsubsection{Exclusion criteria}

In-patients, mothers in labor, very weak patients and speech-impaired mothers were excluded from the study. The weak may require more health services and unable to wait for an interview; while the speech-impaired mothers were not be able to give a verbal answer to the questions asked.

\section{4 Sample size estimation}

Participants were drawn from patients exiting the facility. The sample size was estimated using the Cochran (1963) formula for cross sectional studies $n=\frac{z^{2} p q}{e^{2}}$

Thus, $n=380$ respondents.

This is a non-probability convenience sampling method was used where by the sample is selected based on the availability of respondents who meet all the inclusion criteria was included in the study by the researcher.

\subsection{Sampling procedure}

The researcher used quota sampling method. This is a non-probability sampling method where by the sample is selected from a location convenient for the researcher. When a person with this visible characteristic is identified, the subject is requested to participate in the study; the process continues until you are able to acquire the appropriate number of respondents(15). In this study, the researcher liaised with health workers to identify the pregnant women who had enrolled for their first antenatal visits after gestation of 14 weeks. An ultrasound scan was further performed to confirm the actual weeks of the pregnancy and interviews were carried out on the selected mothers until the appropriate sample size was obtained.

\subsection{Data collection tools}

A researcher-administered questionnaire was used to collect the data. It contained both openended and closed-ended questions.

\subsection{Data analysis and presentation}

The data of the study was analyzed using Microsoft Office Excel 2012 and SPSS 16.0. Presentations are in form of tables, pie-charts and graphs. This was done at all levels using SPSS version 22. Univariate analysis obtained the frequency and distribution of study variables. Bivariate analysis used Chi-square test to determine the association between the two variables. Where $\mathrm{P}<0.05$ were considered significant. Multivariate analysis was performed to control the confounding variables by logistic regression analysis. Variables with $\mathrm{P}<0.05$ at bivariate analysis were put into model to establish factors independently associated late enrolment for ANC.

\subsection{Ethical considerations}

Ethical considerations were taken care including but not limited ethical approval, informed consent and confidentiality 
cognizancejournal.com

Kizito Omona et al, Cognizance Journal of Multidisciplinary Studies, Vol.1, Issue.3, March 2021, pg. 30-44

\section{RESULTS}

\subsection{Socio-demographic Characteristics of Respondents}

Table 1: Univariate analysis of socio-demographic characteristics

\begin{tabular}{|c|c|c|}
\hline Variable & Frequency $(n=380)$ & Percentage $\%$ \\
\hline $\begin{array}{ll}\text { Age } & \\
\text { - } & 15-19 \text { years } \\
\text { - } & 20-24 \text { years } \\
\text { - } & 25-29 \text { years } \\
\text { - } & 30-34 \text { years } \\
& >34 \text { years }\end{array}$ & $\begin{array}{l}102 \\
110 \\
95 \\
56 \\
17\end{array}$ & $\begin{array}{l}26.8 \\
28.9 \\
25.0 \\
14.7 \\
4.5\end{array}$ \\
\hline $\begin{aligned} & \text { Age at Marriage } \\
& \text { - } 15-19 \text { years } \\
& \text { - } 20-24 \text { years } \\
& \text { - } 25-29 \text { years } \\
& \text { - } 30-34 \text { years }\end{aligned}$ & $\begin{array}{l}294 \\
29 \\
30 \\
27\end{array}$ & $\begin{array}{l}77.4 \\
7.6 \\
7.9 \\
7.1\end{array}$ \\
\hline 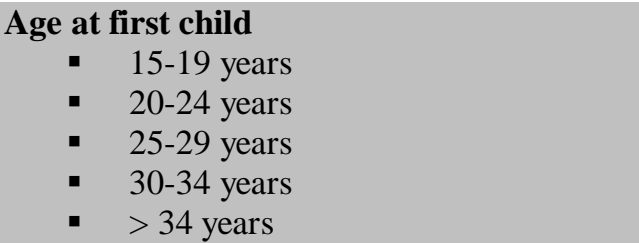 & $\begin{array}{l}321 \\
18 \\
20 \\
20 \\
1\end{array}$ & $\begin{array}{l}84.5 \\
4.7 \\
5.3 \\
5.3 \\
.3\end{array}$ \\
\hline $\begin{aligned} \text { Parity } & \\
\text { - } & \text { One } \\
\text { - } & \text { Two } \\
\text { - } & \text { Three } \\
\text { - } & \text { More than three }\end{aligned}$ & $\begin{array}{l}73 \\
122 \\
111 \\
74\end{array}$ & $\begin{array}{l}19.2 \\
32.1 \\
29.2 \\
19.5\end{array}$ \\
\hline $\begin{array}{ll}\text { Religion } \\
\text { - } \\
\text { - } \text { Patholics } \\
\text { - } \text { Muslims } \\
\text { - Pentecostals }\end{array}$ & $\begin{array}{l}133 \\
109 \\
54 \\
84\end{array}$ & $\begin{array}{l}35.0 \\
28.7 \\
14.2 \\
22.1\end{array}$ \\
\hline $\begin{array}{cl}\text { Marital status } \\
\text { - } \\
\text { - } \text { Single } \\
\text { - } \text { Divorced }\end{array}$ & $\begin{array}{l}136 \\
178 \\
66\end{array}$ & $\begin{array}{l}35.8 \\
46.8 \\
17.4\end{array}$ \\
\hline $\begin{array}{c}\text { Highest Education attained } \\
\text { - No formal education } \\
\text { - } \text { Primary } \\
\text { - } \text { Secondary } \\
\text { Tertiary }\end{array}$ & $\begin{array}{l}106 \\
102 \\
109 \\
63\end{array}$ & $\begin{array}{l}27.9 \\
26.8 \\
28.7 \\
16.6\end{array}$ \\
\hline $\begin{array}{l}\text { Occupation } \\
\text { - Peasant farmer } \\
\text { - Housewife } \\
\text { - } \text { Business woman } \\
\text { - } \text { Civil servant/formal employment } \\
\text { - Informal employment/casual labour }\end{array}$ & $\begin{array}{l}71 \\
94 \\
97 \\
58 \\
60\end{array}$ & $\begin{array}{l}18.7 \\
24.7 \\
25.5 \\
15.3 \\
15.8\end{array}$ \\
\hline $\begin{array}{l}\text { Husband's Highest Education Attained } \\
\text { - No formal education } \\
\text { - Primary } \\
\text { - Secondary } \\
\text { - Tertiary }\end{array}$ & $\begin{array}{l}98 \\
133 \\
108 \\
41\end{array}$ & $\begin{array}{l}25.8 \\
35.0 \\
28.4 \\
10.8\end{array}$ \\
\hline $\begin{array}{c}\text { Husband's occupation } \\
\text { - Peasant farmer } \\
\text { - } \quad \text { Business man }\end{array}$ & $\begin{array}{l}81 \\
191\end{array}$ & $\begin{array}{l}21.3 \\
50.3\end{array}$ \\
\hline
\end{tabular}




\begin{abstract}
- Civil servant/formal employment 79

20.8

- Informal employment/casual labour 29 7.6

Most $28.9 \%$ of the respondents were aged between $20-24$ years, majority $77.4 \%$ of the respondents were aged between 15-19 years at marriage, most $84.5 \%$ of the respondents were aged between 15-19 years at first child, most $32.1 \%$ of the respondents had a parity of two, most $35.0 \%$ of the respondents were Catholics, most $46.8 \%$ of the respondents were single, most $28.7 \%$ of the respondents attained secondary education level, most $25.5 \%$ of the respondents were business women, majority $35.0 \%$ of the respondents had husbands who had attained primary education, and most $50.3 \%$ of the respondent $\mathrm{s}$ whose husbands occupation were businessmen.
\end{abstract}

\title{
3.2 Time of Enrolment for Antenatal Care (ANC) Among Mothers
}

Early enrolment was defined as enrolment on Antenatal care (ANC) within 12 weeks of gestation whereas late enrolment was after 12weeks of gestation

\section{Figure: showing the time at enrollment for ANC}

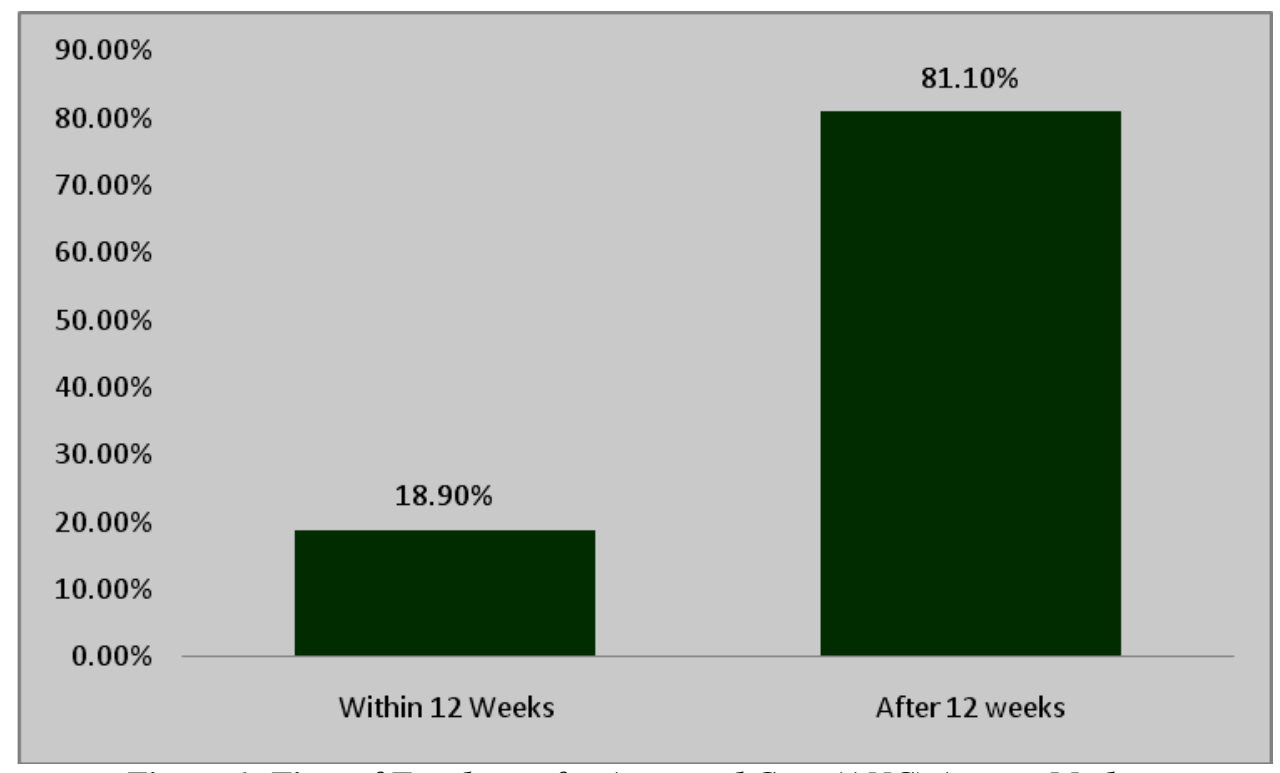

Figure 1: Time of Enrolment for Antenatal Care (ANC) Among Mothers

When the women were asked when they first made their ANC visit with this current pregnancy, majority $81.1 \%$ made their first ANC visit after 12 weeks (Late enrolment) while only $18.9 \%$ made their ANC visit within the first trimester.

\subsection{Socio-demographic Factors Associated with Late Enrolment for ANC}

Analysis of socio-demographic factors associated with late enrolment was done and the result shown in table 2 below 
cognizancejournal.com

Kizito Omona et al, Cognizance Journal of Multidisciplinary Studies, Vol.1, Issue.3, March 2021, pg. 30-44

Table 2: Bivariate analysis of socio-demographic factors associated with late enrollment for ANC

\begin{tabular}{|c|c|c|c|c|c|}
\hline \multirow[t]{2}{*}{ Socio-demographic Variables } & \multicolumn{2}{|c|}{ ANC Enrolment } & \multirow[t]{2}{*}{ Total } & \multirow[t]{2}{*}{$\square^{2}$} & \multirow[t]{2}{*}{ P-value } \\
\hline & Late & Early & & & \\
\hline $\begin{array}{l}\text { Age } \\
\text { - } 15-19 \text { years } \\
\text { - } 20-24 \text { years } \\
\text { - } 30-34 \text { years } \\
\text { - }>34 \text { years } \\
\end{array}$ & $\begin{array}{l}81(26.3 \%) \\
88(28.6 \%) \\
75(24.4 \%) \\
49(15.9 \%) \\
15(4.9 \%)\end{array}$ & $\begin{array}{l}21(29.2 \%) \\
22(30.6 \%) \\
20(27.8 \%) \\
7(9.7 \%) \\
2(2.8 \%)\end{array}$ & $\begin{array}{l}102 \\
110 \\
95 \\
56 \\
17\end{array}$ & $\begin{array}{l}2.619 \\
\mathrm{Df}=4\end{array}$ & .623 \\
\hline $\begin{array}{c}\text { Age at Marriage } \\
: \quad 15-19 \text { years } \\
: \quad 20-24 \text { years } \\
\text { : } 25-29 \text { years } \\
\text { : } 30-34 \text { years }\end{array}$ & $\begin{array}{l}242(78.6 \%) \\
26(8.4 \%) \\
23(7.5 \%) \\
17(5.5 \%) \\
\end{array}$ & $\begin{array}{l}52(72.2 \%) \\
3(4.2 \%) \\
7(9.7 \%) \\
10(13.9 \%)\end{array}$ & $\begin{array}{l}294 \\
29 \\
30 \\
27\end{array}$ & $\begin{array}{l}7.830 \\
\mathrm{Df}=3\end{array}$ & $.050 *$ \\
\hline $\begin{array}{c}\text { Age at first child } \\
\text { : } 15-19 \text { years } \\
\text { - } 20-24 \text { years } \\
\text {. } 25-29 \text { years } \\
\text { - } 34 \text { years } \\
\end{array}$ & $\begin{array}{l}256(83.1 \%) \\
15(4.9 \%) \\
19(6.2 \%) \\
17(5.5 \%) \\
1(.3 \%) \\
\end{array}$ & $\begin{array}{l}65(90.3 \%) \\
3(4.2 \%) \\
1(1.4 \%) \\
3(4.2 \%) \\
0(.0 \%)\end{array}$ & $\begin{array}{l}321 \\
18 \\
20 \\
20 \\
1\end{array}$ & $\begin{array}{l}3.385 \\
\mathrm{Df}=4\end{array}$ & .496 \\
\hline $\begin{aligned} & \text { Parity } \\
& \text { - } \text { One } \\
& \text { - } \text { Thro } \\
& \text { - } \text { More than three } \\
&\end{aligned}$ & $\begin{array}{l}56(18.2 \%) \\
85(27.6 \%) \\
103(33.4 \%) \\
64(20.8 \%) \\
\end{array}$ & $\begin{array}{l}17(23.6 \%) \\
37(51.4 \%) \\
8(11.1 \%) \\
10(13.9 \%)\end{array}$ & $\begin{array}{l}73 \\
122 \\
111 \\
74 \\
\end{array}$ & $\begin{array}{l}22.569 \\
D f=3\end{array}$ & $.000^{*}$ \\
\hline $\begin{aligned} & \text { Religion } \\
& \text { : } \text { Catholics } \\
& \text { : } \text { Protestants } \\
& \text { - } \text { Pentims } \\
&\end{aligned}$ & $\begin{array}{l}110(35.7 \%) \\
76(24.7 \%) \\
46(14.9 \%) \\
76(24.7 \%) \\
\end{array}$ & $\begin{array}{l}23(31.9 \%) \\
33(45.8 \%) \\
8(11.1 \%) \\
8(11.1 \%) \\
\end{array}$ & $\begin{array}{l}133 \\
109 \\
54 \\
84 \\
\end{array}$ & $\begin{array}{l}14.802 \\
D f=3\end{array}$ & $.002 *$ \\
\hline $\begin{array}{cl}\text { Marital status } \\
\text { - } & \text { Married } \\
\text { - } & \text { Single } \\
\text { - } & \text { Divorced } \\
\end{array}$ & $\begin{array}{l}116(37.7 \%) \\
142(46.1 \%) \\
50(16.2 \%)\end{array}$ & $\begin{array}{l}20(27.8 \%) \\
36(50.0 \%) \\
16(22.2 \%)\end{array}$ & $\begin{array}{l}136 \\
178 \\
66 \\
\end{array}$ & $\begin{array}{l}2.987 \\
\mathrm{Df}=2\end{array}$ & .225 \\
\hline $\begin{aligned} & \text { Highest Education attained } \\
& \text { - } \text { No formal education } \\
& \text { - } \text { Primary } \\
& \text { - } \text { Tecondary } \\
&\end{aligned}$ & $\begin{array}{l}89(28.9 \%) \\
89(28.9 \%) \\
82(26.6 \%) \\
49(15.9 \%)\end{array}$ & $\begin{array}{l}17(23.6 \%) \\
14(19.4 \%) \\
27(37.5 \%) \\
14(19.4 \%) \\
\end{array}$ & $\begin{array}{l}106 \\
103 \\
109 \\
63 \\
\end{array}$ & $\begin{array}{l}5.242 \\
\mathrm{Df}=3\end{array}$ & .155 \\
\hline $\begin{aligned} & \text { Occupation } \\
& \text { : } \text { Peasant farmer } \\
& \text { : } \text { Housewife } \\
& \text { - } \text { Civil sess womant/formal employment } \\
& \text { Informal employment/casual labor }\end{aligned}$ & $\begin{array}{l}54(17.5 \%) \\
80(26.0 \%) \\
74(24.0 \%) \\
45(14.6 \%) \\
55(17.9 \%)\end{array}$ & $\begin{array}{l}17(23.6 \%) \\
14(19.4 \%) \\
23(31.9 \%) \\
13(18.1 \%) \\
5(6.9 \%)\end{array}$ & $\begin{array}{l}71 \\
94 \\
97 \\
58 \\
60\end{array}$ & $\begin{array}{l}8.449 \\
\mathrm{Df}=4\end{array}$ & .076 \\
\hline $\begin{aligned} & \text { Husband's Highest Education Attained } \\
& \text { - } \text { No formal education } \\
& \text { - Primary } \\
& \text { - } \\
& \text { Secondary } \\
& \text { Tertiary }\end{aligned}$ & $\begin{array}{l}73(23.7 \%) \\
119(38.6 \%) \\
82(26.6 \%) \\
34(11.0 \%)\end{array}$ & $\begin{array}{l}25(34.7 \%) \\
14(19.4 \%) \\
26(36.1 \%) \\
7(9.7 \%)\end{array}$ & $\begin{array}{l}98 \\
133 \\
108 \\
41 \\
\end{array}$ & $\begin{array}{l}10.832 \\
D f=3\end{array}$ & $.013 *$ \\
\hline
\end{tabular}


cognizancejournal.com

Kizito Omona et al, Cognizance Journal of Multidisciplinary Studies, Vol.1, Issue.3, March 2021, pg. 30-44

\begin{tabular}{|c|l|l|l|l|l|}
\hline Husband's occupation & $61(19.8 \%)$ & $20(27.8 \%)$ & 81 & 3.555 & .314 \\
- Peasant farmer & $161(52.3 \%)$ & $30(41.7 \%)$ & 191 & Df=3 & \\
- Business man & $64(20.8 \%)$ & $15(20.8 \%)$ & 79 & & \\
- Informal employment/casual labor & $22(7.1 \%)$ & $7(9.7 \%)$ & 29 & & \\
\hline
\end{tabular}

$\square^{2}=$ Chi square, $\mathbf{D f}=$ Degree of freedom

Among the socio-demographic factors, having age at marriage $\left(\square^{2}=7.830, \boldsymbol{p}=\mathbf{0 . 0 5 0}\right)$, parity $\left(\square^{2}=22.569, \boldsymbol{p}=\mathbf{0 . 0 0 0}\right)$, religion $\left(\square^{2}=14.802, \boldsymbol{p}=\mathbf{0 . 0 0 2}\right)$, and husbands education level $\left(\square^{2}=\right.$ $10.832, \boldsymbol{p}=\mathbf{0 . 0 1 3}$ ) were significantly associated with late enrolment for ANC.

\subsection{Knowledge of Mothers on antenatal care services}

Bivariate analysis of knowledge of women on ANC services associated with late enrolment for ANC was done. See table 3 below

Table 3: Bivariate analysis of Knowledge of women on ANC services

\begin{tabular}{|c|c|c|c|c|c|}
\hline \multirow[t]{2}{*}{ Knowledge Variables } & \multicolumn{2}{|c|}{ ANC Enrolment } & \multirow[t]{2}{*}{ Total } & \multirow[t]{2}{*}{$\square^{2}$} & \multirow[t]{2}{*}{ P-value } \\
\hline & Late & Early & & & \\
\hline $\begin{array}{l}\text { What is antenatal care? } \\
\text { - Medical care of a pregnant woman during } \\
\text { pregnancy. } \\
\text { - Taking of medication by pregnant women } \\
\text { - I don't know }\end{array}$ & $\begin{array}{l}142(46.1 \%) \\
121(39.3 \%) \\
45(14.6 \%)\end{array}$ & $\begin{array}{l}14(19.4 \%) \\
41(56.9 \%) \\
17(23.6 \%)\end{array}$ & $\begin{array}{l}156 \\
162 \\
62\end{array}$ & $\begin{array}{l}17.270 \\
\mathrm{Df}=2\end{array}$ & $.000 *$ \\
\hline $\begin{array}{l}\text { When is the right time to seek antenatal care? } \\
\text { - After } 14 \text { weeks of pregnancy } \\
\text { - After } 28 \text { weeks of pregnancy } \\
\text { - After } 32 \text { weeks of pregnancy } \\
\text { - Immediately }\end{array}$ & $\begin{array}{l}79(25.6 \%) \\
121(39.3 \%) \\
54(17.5 \%) \\
54(17.5 \%)\end{array}$ & $\begin{array}{l}15(20.8 \%) \\
22(30.6 \%) \\
29(40.3 \%) \\
6(8.3 \%)\end{array}$ & $\begin{array}{l}94 \\
143 \\
83 \\
60\end{array}$ & $\begin{array}{l}18.679 \\
\mathrm{Df}=3\end{array}$ & $.000 *$ \\
\hline $\begin{array}{l}\text { Do you think it is important to book early for } \\
\text { antenatal care? } \\
\text { - Yes } \\
\text { - No }\end{array}$ & $\begin{array}{l}98(31.8 \%) \\
210(68.2 \%)\end{array}$ & $\begin{array}{l}24(33.3 \%) \\
48(66.7 \%) \\
\end{array}$ & $\begin{array}{l}122 \\
258 \\
\end{array}$ & .061 & .453 \\
\hline $\begin{array}{l}\text { If yes, give at least } 2 \text { benefits of early booking by } \\
\text { pregnant mothers } \\
\text { - Early detection, treatment and care of } \\
\text { infections } \\
\text { - Improved neonatal health }\end{array}$ & $\begin{array}{l}48(49.0 \%) \\
50(51.0 \%)\end{array}$ & $\begin{array}{l}16(66.7 \%) \\
8(33.3 \%)\end{array}$ & 64 & $\begin{array}{l}2.418 \\
\mathrm{Df}=1\end{array}$ & .092 \\
\hline $\begin{array}{l}\text { What is the recommended number of times to } \\
\text { attend antenatal care among pregnant mothers? } \\
\text { - times } \\
\text { - } 5 \text { times } \\
\text { - } 4 \text { times }\end{array}$ & $\begin{array}{l}123(39.9 \%) \\
84(27.3 \%) \\
101(32.8 \%)\end{array}$ & $\begin{array}{l}8(11.1 \%) \\
50(69.4 \%) \\
14(19.4 \%)\end{array}$ & $\begin{array}{l}131 \\
134 \\
115\end{array}$ & $\begin{array}{l}46.932 \\
\mathrm{Df}=2\end{array}$ & $.000 *$ \\
\hline $\begin{array}{l}\text { Who makes your decisions to seek health care } \\
\text { services like antenatal care? } \\
\text { - I make the decision } \\
\text { - My partner makes the decision } \\
\text { - My mother-in-law } \\
\text { - We both make the decision }\end{array}$ & $\begin{array}{l}86(27.9 \%) \\
103(33.4 \%) \\
75(24.4 \%) \\
44(14.3 \%)\end{array}$ & $\begin{array}{l}26(36.1 \%) \\
26(36.1 \%) \\
12(16.7 \%) \\
8(11.1 \%)\end{array}$ & $\begin{array}{l}112 \\
129 \\
87 \\
52\end{array}$ & $\begin{array}{l}3.385 \\
\mathrm{Df}=3\end{array}$ & .336 \\
\hline $\begin{array}{l}\text { Fansidar for prevention of malaria in pregnant } \\
\text { should be given to pregnant women every month } \\
\text { [from } 14 \text { weeks] until she delivers } \\
\text { - True }\end{array}$ & $189(61.4 \%)$ & $40(55.6 \%)$ & 229 & .822 & .219 \\
\hline
\end{tabular}


cognizancejournal.com

Kizito Omona et al, Cognizance Journal of Multidisciplinary Studies, Vol.1, Issue.3, March 2021, pg. 30-44

\begin{tabular}{|l|l|l|l|l|l|}
\hline \multicolumn{1}{|l|}{ False } & $119(38.6 \%)$ & $32(44.4 \%)$ & 151 & $\mathrm{Df}=1$ & \\
\hline $\begin{array}{l}\text { Screening blood for infections (HIV and Syphilis) } \\
\text { should be carried out during antenatal care check- } \\
\text { up. }\end{array}$ & $164(53.2 \%)$ & $47(65.3 \%)$ & 211 & 3.421 & $\mathbf{. 0 4 2 *}$ \\
- True & $144(46.8 \%)$ & $25(34.7 \%)$ & 169 & $\mathrm{Df}=1$ & \\
\hline $\begin{array}{l}\text { Supplementation of iron and folic acid are good for } \\
\text { mother and fetus }\end{array}$ & & & & & \\
$-\quad$ True & $147(47.7 \%)$ & $28(38.9 \%)$ & 175 & 1.835 & .110 \\
$-\quad$ False & $161(52.3 \%)$ & $44(61.1 \%)$ & 205 & $\mathrm{Df}=1$ & \\
\hline
\end{tabular}

$\square^{2}=$ Chi square, $\mathbf{D f}=$ Degree of freedom

Meaning of Antenatal care $\left(\square^{2}=17.270\right.$, p-value of 0.000$)$, knowledge of the right time to seek antenatal care $\left(\square^{2}=18.679\right.$, p-value of 0.000$)$, knowledge of the recommended times of antenatal attendance $\left(\square^{2}=46.932\right.$, p-value of 0.000$)$ and knowledge the screening blood for infections $\left(\square^{2}=3.421\right.$, p-value of 0.042$)$ were the knowledge of antenatal care services associated with late enrolment.

\subsection{Attitudes of Mothers towards ANC services}

The attitudes of mothers towards ANC services was analysed for any association with late ANC visit and the result are tabulated below

Table 4: Bivariate Analysis of Attitudes of Mothers Towards ANC Enrolment

\begin{tabular}{|c|c|c|c|c|c|}
\hline \multirow[t]{2}{*}{ Attitude Variables } & \multicolumn{2}{|c|}{ ANC Enrolment } & \multirow[t]{2}{*}{ Total } & \multirow[t]{2}{*}{$\square^{2}$} & \multirow{2}{*}{$\begin{array}{l}\text { P- } \\
\text { value }\end{array}$} \\
\hline & Late & Early & & & \\
\hline $\begin{array}{l}\text { It is necessary to register during pregnancy } \\
\text { for antenatal check up } \\
\text { - Strongly Agree } \\
\text { - Agree } \\
\text { - Disagree } \\
\text { - Strongly Disagree }\end{array}$ & $\begin{array}{l}55(17.9 \%) \\
88(28.6 \%) \\
93(30.2 \%) \\
72(23.4 \%)\end{array}$ & $\begin{array}{l}16(22.2 \%) \\
26(36.1 \%) \\
21(29.2 \%) \\
9(12.5 \%)\end{array}$ & $\begin{array}{l}71 \\
114 \\
114 \\
81\end{array}$ & $\begin{array}{l}4.960 \\
D f=3\end{array}$ & .175 \\
\hline $\begin{array}{l}\text { It is importance for a pregnant woman to } \\
\text { have early antenatal booking } \\
\text { - Strongly Agree } \\
\text { - Agree } \\
\text { - Disagree } \\
\text { - Strongly Disagree }\end{array}$ & $\begin{array}{l}74(24.0 \%) \\
91(29.5 \%) \\
77(25.0 \%) \\
66(21.4 \%)\end{array}$ & $\begin{array}{l}9(12.5 \%) \\
7(9.7 \%) \\
24(33.3 \%) \\
32(44.4 \%)\end{array}$ & $\begin{array}{l}83 \\
98 \\
101 \\
98\end{array}$ & $\begin{array}{l}25.953 \\
\mathrm{Df}=3\end{array}$ & $.000 *$ \\
\hline $\begin{array}{l}\text { Antenatal care follow-up is good to monitor } \\
\text { mother's and fetus's health } \\
\text { - Strongly Agree } \\
\text { - Agree } \\
\text { - Disagree } \\
\text { - Strongly Disagree }\end{array}$ & $\begin{array}{l}69(22.4 \%) \\
103(33.4 \%) \\
104(33.8 \%) \\
32(10.4 \%)\end{array}$ & $\begin{array}{l}4(5.6 \%) \\
35(48.6 \%) \\
31(43.1 \%) \\
2(2.8 \%)\end{array}$ & $\begin{array}{l}73 \\
138 \\
135 \\
34\end{array}$ & $\begin{array}{l}17.516 \\
\mathrm{Df}=3\end{array}$ & $.001 *$ \\
\hline $\begin{array}{l}\text { Antenatal care is very vital for the health of } \\
\text { the mothers and the baby } \\
\text { - Strongly Agree } \\
\text { - Agree } \\
\text { - Disagree } \\
\text { - Strongly Disagree }\end{array}$ & $\begin{array}{l}50(16.2 \%) \\
110(35.7 \%) \\
68(22.1 \%) \\
80(26.0 \%)\end{array}$ & $\begin{array}{l}24(33.3 \%) \\
12(16.7 \%) \\
12(16.7 \%) \\
24(33.3 \%)\end{array}$ & $\begin{array}{l}74 \\
122 \\
80 \\
104\end{array}$ & $\begin{array}{l}17.324 \\
\mathrm{Df}=3\end{array}$ & $.001 *$ \\
\hline $\begin{array}{l}\text { I prefer to go to TBA/ instead of going for } \\
\text { Antenatal care } \\
\text { - Strongly Agree } \\
\text { - Agree } \\
\text { - Disagree } \\
\end{array}$ & $\begin{array}{l}49(15.9 \%) \\
125(40.6 \%) \\
62(20.1 \%)\end{array}$ & $\begin{array}{l}12(16.7 \%) \\
16(22.2 \%) \\
26(36.1 \%) \\
\end{array}$ & $\begin{array}{l}61 \\
141 \\
88 \\
\end{array}$ & $\begin{array}{l}11.825 \\
\mathrm{Df}=3\end{array}$ & $.008 *$ \\
\hline
\end{tabular}


cognizancejournal.com

Kizito Omona et al, Cognizance Journal of Multidisciplinary Studies, Vol.1, Issue.3, March 2021, pg. 30-44

\begin{tabular}{|c|c|c|c|c|c|}
\hline - $\quad$ Strongly Disagree & $72(23.4 \%)$ & $18(25.0 \%)$ & 90 & & \\
\hline $\begin{array}{l}\text { My religious beliefs does not allows ANC } \\
\text { - Strongly Agree } \\
\text { - Agree } \\
\text { - } \text { Disagree } \\
\text { - Strongly Disagree }\end{array}$ & $\begin{array}{l}62(20.1 \%) \\
86(27.9 \%) \\
72(23.4 \%) \\
88(28.6 \%)\end{array}$ & $\begin{array}{l}10(13.9 \%) \\
18(25.0 \%) \\
26(36.1 \%) \\
18(25.0 \%)\end{array}$ & $\begin{array}{l}72 \\
104 \\
98 \\
106\end{array}$ & $\begin{array}{l}5.318 \\
D f=3\end{array}$ & .150 \\
\hline $\begin{array}{l}\text { Am willing to attend ANC services when am } \\
\text { pregnant } \\
\text { - } \text { Strongly Agree } \\
\text { - } \text { Agree } \\
\text { - } \text { Disagree } \\
\end{array}$ & $\begin{array}{l}36(11.7 \%) \\
100(32.5 \%) \\
83(26.9 \%) \\
89(28.9 \%)\end{array}$ & $\begin{array}{l}18(25.0 \%) \\
32(44.4 \%) \\
14(19.4 \%) \\
8(11.1 \%)\end{array}$ & $\begin{array}{l}54 \\
132 \\
97 \\
97\end{array}$ & $\begin{array}{l}18.206 \\
\mathrm{Df}=3\end{array}$ & $.000 *$ \\
\hline $\begin{array}{l}\text { My family and the society support me to } \\
\text { follow ANC services } \\
\text { - Strongly Agree } \\
\text { - Agree } \\
\text { - Disagree } \\
\text { - Strongly Disagree }\end{array}$ & $\begin{array}{l}60(19.5 \%) \\
62(20.1 \%) \\
146(47.4 \%) \\
40(13.0 \%)\end{array}$ & $\begin{array}{l}24(33.3 \%) \\
14(19.4 \%) \\
14(19.4 \%) \\
20(27.8 \%)\end{array}$ & $\begin{array}{l}84 \\
76 \\
160 \\
60\end{array}$ & $\begin{array}{l}23.999 \\
\mathrm{Df}=3\end{array}$ & $.000 *$ \\
\hline $\begin{array}{l}\text { I feel sulphadoxinepyremethamine (SP) } \\
\text { treatment are important for prevention of } \\
\text { Malaria during their pregnancy } \\
\text { - Strongly Agree } \\
\text { - Agree } \\
\text { - Disagree } \\
\text { - Strongly Disagree }\end{array}$ & $\begin{array}{l}64(20.8 \%) \\
80(26.0 \%) \\
120(39.0 \%) \\
44(14.3 \%)\end{array}$ & $\begin{array}{l}17(23.6 \%) \\
3(4.2 \%) \\
22(30.6 \%) \\
30(41.7 \%)\end{array}$ & $\begin{array}{l}81 \\
83 \\
142 \\
74\end{array}$ & $\begin{array}{l}36.496 \\
\mathrm{Df}=3\end{array}$ & $.000 *$ \\
\hline $\begin{array}{l}\text { By screening for HIV means that I have } \\
\text { understanding of HIV transmission from the } \\
\text { mother to child in the community. } \\
\text { - Strongly Agree } \\
\text { - Agree } \\
\text { - Disagree } \\
\text { - Strongly Disagree }\end{array}$ & $\begin{array}{l}35(11.4 \%) \\
123(39.9 \%) \\
104(33.8 \%) \\
46(14.9 \%)\end{array}$ & $\begin{array}{l}18(25.0 \%) \\
30(41.7 \%) \\
16(22.2 \%) \\
8(11.1 \%)\end{array}$ & $\begin{array}{l}53 \\
153 \\
120 \\
54 \\
\end{array}$ & $\begin{array}{l}10.887 \\
\mathrm{Df}=3\end{array}$ & $.012 *$ \\
\hline
\end{tabular}

]$^{2}=$ Chi square, $\mathbf{D f}=$ Degree of freedom

Importance for a pregnant woman to have early antenatal booking $\left(\square^{2}=25.953, \mathrm{p}=0.000\right)$, Antenatal care follow-up is good to monitor mother's and fetus's health $\left(\square^{2}=17.516, \mathrm{p}=\right.$ $0.001)$, antenatal care is very vital for the health of the mothers and the baby $\left(\square^{2}=17.324\right.$, $\mathrm{p}=0.001)$, preference to go to TBA/ instead of going for Antenatal care $\left(\square^{2}=11.825\right.$, $\mathrm{p}=0.008)$, willing to attend ANC services when pregnant $\left(\square^{2}=18.206, \mathrm{p}=0.000\right)$, feeling of family and the society support to follow ANC services $\left(\square^{2}=23.999, p=0.000\right)$, feeling that sulphadoxinepyremethamine (SP) treatment are important for prevention of Malaria during their pregnancy $\left(\square^{2}=36.496, p=0.000\right)$ and feeling that by screening for HIV means that I have understanding of HIV transmission from the mother to child in the community $\left(\square^{2}=10.887, \mathrm{p}=0.012\right)$ were associated with late enrolment for ANC.

Table 5: Multivariate analysis of factors associated with late enrollment for ANC

\begin{tabular}{|l|l|l|}
\hline Variables & $\begin{array}{l}\text { Adjusted OR (AOR), } \\
\text { 95\% CI (L-U) }\end{array}$ & p-value \\
\hline Religion & 1.0 & \\
- Catholics & $9.943(1.089-90.821)$ & $.042 *$ \\
- Muslims & $.083(.002-4.095)$ & .211 \\
\hline & $.714(.044-11.706)$ & .814 \\
\hline
\end{tabular}


cognizancejournal.com

Kizito Omona et al, Cognizance Journal of Multidisciplinary Studies, Vol.1, Issue.3, March 2021, pg. 30-44

\begin{tabular}{|c|c|c|}
\hline $\begin{array}{l}\text { Knowledge on right time to seek first antenatal care } \\
\text { - After } 14 \text { weeks of pregnancy } \\
\text { - After } 28 \text { weeks of pregnancy } \\
\text { - After } 32 \text { weeks of pregnancy } \\
\text { - Immediately }\end{array}$ & $\begin{array}{l}1.0 \\
3.019(1.001-6.342) \\
2.214(.154-31.912) \\
.007(.001-.093)\end{array}$ & $\begin{array}{l}.000 * \\
.559 \\
.007 *\end{array}$ \\
\hline $\begin{array}{l}\text { Recommended number of ANC visits } \\
\text { - } \quad \text { times } \\
\text { - } 5 \text { times } \\
\text { - } 4 \text { times }\end{array}$ & $\begin{array}{l}1.0 \\
.556(.355-5.684) \\
.777(.294-.890)\end{array}$ & $\begin{array}{l}.169 \\
.001 *\end{array}$ \\
\hline $\begin{array}{l}\text { Antenatal care follow-up is good to monitor mother's and } \\
\text { fetus's health } \\
\text { - Strongly Agree } \\
\text { - Agree } \\
\text { - } \text { Strogree } \\
\end{array}$ & $\begin{array}{l}1.0 \\
12.327(.036-49.952) \\
33.189(2.639-42.419) \\
36.348(4.365-92.711) \\
\end{array}$ & $\begin{array}{l}.391 \\
.019 * \\
.009 *\end{array}$ \\
\hline $\begin{array}{l}\text { I prefer to go to TBA instead of going for Antenatal care } \\
\text { - } \text { Strongly Agree } \\
\text { - } \text { Agree } \\
\text { - } \text { Disagree } \\
\end{array}$ & $\begin{array}{l}1.0 \\
1.015(.007-2.026) \\
.216(.106-.989) \\
.232(.112-6.056) \\
\end{array}$ & $\begin{array}{l}.051 \\
.049 * \\
.105\end{array}$ \\
\hline $\begin{array}{l}\text { Am willing to attend ANC services when am pregnant } \\
\text { - } \text { Strongly Agree } \\
\text { - } \text { Agree } \\
\text { - } \text { Disagree } \\
\end{array}$ & $\begin{array}{l}1.0 \\
6.010(2.005-15.026) \\
8.021(2.008-12.089) \\
10.033(4.009-.30 .100)\end{array}$ & $\begin{array}{l}.000 * \\
.000 * \\
.000 *\end{array}$ \\
\hline $\begin{array}{l}\text { My family and the society support me to follow ANC services } \\
\text { - Strongly Agree } \\
\text { - Agree } \\
\text { - } \text { Disagree } \\
\text { - Strongly Disagree }\end{array}$ & $\begin{array}{l}1.0 \\
1.204(.012-3.614) \\
4.037(1.013-5.352) \\
1.209(.010-4.594)\end{array}$ & $\begin{array}{l}.279 \\
.013 * \\
.321\end{array}$ \\
\hline $\begin{array}{l}\text { I feel sulphadoxinepyremethamine (SP) treatment are } \\
\text { important for prevention of Malaria during their pregnancy } \\
\text { - Strongly Agree } \\
\text { - Agree } \\
\text { - Disagree } \\
\text { - Strongly Disagree }\end{array}$ & $\begin{array}{l}1.0 \\
2.111(1.025-7.068) \\
3.032(1.008-10.010) \\
2.035(1.002-9.708)\end{array}$ & $\begin{array}{l}.002 * \\
.000 * \\
.029 *\end{array}$ \\
\hline
\end{tabular}

Respondents who were Protestants/Pentecostal were 9.943 times more likely to have late enrolment compared to those who were Catholics. Respondents who were knew that the right time to seek first ANC is after 28 weeks were 3.019 times more likely to enrol late compared to those knew that the right time to seek first ANC was after 14 weeks while those who knew that the right time to seek first ANC were 0.007 times less likely to enrol late.

Respondents who knew that the recommended number of ANC visits were 4 times were 0.777 times less likely to enrol late compared to those who knew that the recommended ANC visits are two times

Respondents who disagreed and strongly disagreed that ANC follow up is good to monitor mothers and fetus health were 33.189 and 36.348 times more likely to have late ANC enrolment.

Respondents who disagreed that they prefer to go to TBA instead of going for ANC were 0.216 times less likely to have late enrollment for ANC compared to those who strongly agreed that they prefer to go to TBA instead of going for ANC.

Respondents who agreed, disagreed and strongly disagreed that they were willing to attend ANC services when they are pregnant were 6.010, 8.021 and 10.033 times more likely to have late ANC enrolment respectively. 


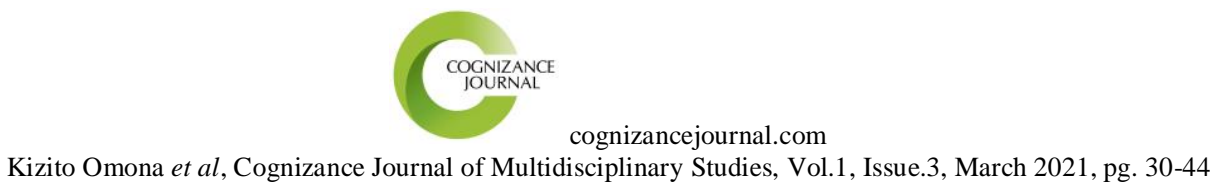

Respondents who disagreed that their family and society support them to follow ANC services were 4.037 times more likely to have late ANC enrolment.

Respondents who agreed, disagreed and strongly disagreed that they feel sulphadoxinepyremethamine (SP) treatment are important for prevention of Malaria during their pregnancy were 2.111, 3.032 and 2.035 times more likely to have late ANC enrolment.

\section{DISCUSSIONS}

\subsection{Late enrolment for ANC}

The study found that the prevalence of late enrolment was $81.1 \%$ visit among the study respondents with only $18.9 \%$ enrolled early for ANC. This is probably because the women are not well-informed on importance of timely first ANC visit since there is no formal education and no health extension workers affected the timing of 1st ANC in the community. This is slightly above the prevalence by some scholars (16) which found that $17.4 \%$ of the mother reported for the first ANC within the recommended time. Finding here is similar to one study (14) which found the prevalence at $9.7 \%$ of the respondents had their first ANC visit at the recommended time frame. A related study (17) found that over $40 \%$ of pregnant women do not initiate $1^{\text {st }}$ ANC visit in the first trimester. The difference in the study could probably be the difference in study setting. This implies that there is need to give an advice, improve economic capacity and avoid unplanned pregnancy through family planning.

\subsection{Socio-demographic factors}

In this study, religious of the participants was significantly associated with late enrollment for ANC; Protestants/Pentecostals had high chances of late enrollment. This is similar to a study by (18) who found that the timing and frequency of antenatal visits were significantly associated with religious differences. Similarly(14), it was found that cultural beliefs and religious ideas about pregnancy can influence women to make a decision about when to start ANC early or not to attend at all.

In the study, education level of the respondents was found to be associated with late enrolment for ANC. This is probably because educated women are exposed to information regarding ANC and its importance to the woman and the child since they can read some of the information besides being able to work and support themselves and more educated mothers had better income, income increases the ability to pay for health care services, transportation, and other indirect costs. This is consistent with one scholar(18) who noted that timing and frequency of antenatal visits were significantly associated with education of the mother. Similarly(19), one stated that underutilization of ANC was due to lack of education, pregnant mothers with no formal education (illiterate women) attended late ANC than women who had formal education. This implies that women should be empowered and given equal opportunity on education in communities

\subsection{Knowledge on ANC services}

The study found that knowledge about the number of visit was associated with late enrolment; knowing that there were four recommended ANC was associated with reduced chances of late enrolment. This is in line with a study (20), which showed that many of the mother's income were low, so they failed to raise money for transport, lunch while on ANC visit lacked what to put on like maternity dresses and knickers and lack of the required number of visit. In addition (14), it was also shown that only $9.7 \%$ of the respondents had 


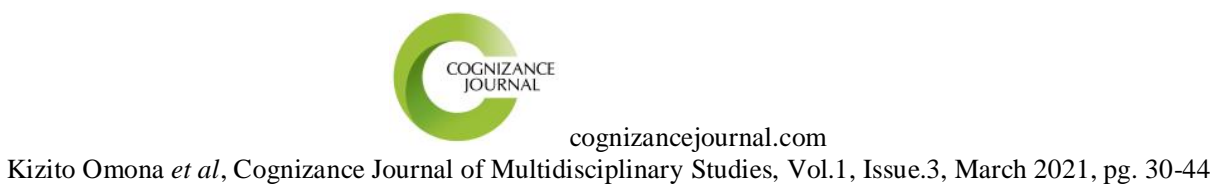

their first ANC visit at the recommended time frame those most of them did not know their gestation age.

The study found that knowledge that one should start ANC immediately was associated with reduced chance of late enrolment to ANC. This is supported by a related study (17) which found that over $40 \%$ of pregnant women do not initiate $1^{\text {st }}$ ANC visit in the first trimester largely due to lack of correct knowledge of the recommended ANC schedule.

\subsection{Attitude towards ANC services}

The study found that believing in TBA as an alternative was associated with increased chance of late uptake of ANC services. This is in line with a study (12), which observed that in many countries, TBAs are an important source of social and cultural support to women during childbirth, due to economic constraints and difficulty in posting trained professionals to rural areas forcing many women continue to deliver with TBAs which prevent them from access ANC services from qualified health professionals. This is also Delivery also was conducted at home or TBA because of fear of mode of delivery at hospital, encouraged by mother or mother in law, husband's decision, lack of problems during pregnancy and very few being interested in delivering at health facility. This means that there is inadequate sensitization done specifically in these rural areas to empower pregnant women and their husbands as to improve timing of 1 st ANC attendance.

In the study, feeling of having support from family and society to follow ANC services was associated with reduced chance of late ANC visits. This is in line with a study by another scholar(18) who noted that some mothers enrolled for ANC late because they had not gotten permission from their husbands. In addition(20), it was also found that out late recognition of pregnancy and not being supported by the husband or partner were identified as factors associated with a late antenatal care booking and (21) also found that lack of male partner support during antenatal period are associated with delayed booking for antenatal care.

\section{CONCLUSIONS}

The general proportion of late enrolment for ANC was high at $81.1 \%$. Being protestants/Pentecostals was associated with high occurrence of late enrolment for ANC. Knowledge that ANC should start immediately and that the recommended number of ANC visits should be four were associated with low chance of late enrolment. While knowing that ANC should start after 28 weeks was positively associated with late enrolment. Having strong agreement that Antenatal care follow-up is good to monitor mother's and fetus's health, willing to attend ANC services when am pregnant, having support from family and society to follow ANC services and having feelings that sulphadoxinepyremethamine (SP) treatment are important for prevention of Malaria during their pregnancy were negatively associated with late enrolment for ANC while having strong agreement of preferring TBA instead of going for ANC was associated with high chance of late enrolment for ANC

\section{RECOMMENDATIONS}

The study, therefore, recommends that the District stake holders, District health office and community leaders should sensitize the community on the importance of keeping the girl child at school up to the time of completion of their studies. Health care providers are encouraged to give enough information during health education on the importance of timing of 1 st antenatal care, number of visits and encourage mothers deliver from health facility and 


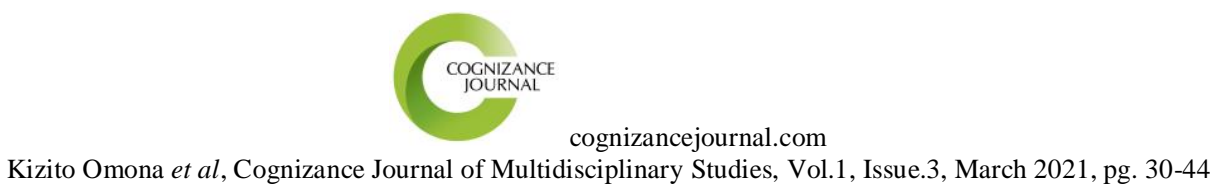

future qualitative studies exploring the enablers and factors to early antenatal care booking in other public health facilities within Masindi District.

\section{ACKNOWLEDGEMENTS}

We would like to thank the pregnant mothers who participated in the interviews and we acknowledge the following for their contribution towards this research work: to the entire staff of Masindi hospital and lastly the Staff at Uganda Martyrs University, Faculty of Health Sciences.

\section{DECLARATION}

The authors declare that they had no conflict of interest.

\section{REFERENCES}

1. WHO recommendations on antenatal care for a positive pregnancy experience. 2016.

2. Manyeh AK, Amu A, Williams J, Gyapong M. Factors associated with the timing of antenatal clinic attendance among first- time mothers in rural southern Ghana. 2020;0:1-7.

3. Organisation world H. No Title [Internet]. Maternal Mortality. 2019 [cited 2020 May 20]. Available from: https://www.who.int/news-room/fact-sheets/detail/maternalmortality.

4. Finlayson K. What matters to women: a systematic scoping review to identify the processes and outcomes of antenatal care provision that are important to healthy pregnant women. 2015;529-39.

5. Carroli G, Rooney C. How effective is antenatal care in preventing maternal mortality and serious morbidity? An overview of the evidence. 2001;15.

6. UNICEF. No Title [Internet]. Antenatal Care. 2020. Available from: https://www.unicef.org/uganda/key-practice-antenatal-care.

7. Lincetto O, Mothebesoane-anoh S, Gomez P, Munjanja S. Antenatal Care. :51-62.

8. Bale JR, Stoll BJ, Lucas AO. Improving Birth Outcomes, Meeting the Challenge in the Developing World.2003.

9. Maternal U, Vision H, Evidence A, Approaches S. Ending Preventable Maternal Mortality: USAID Maternal Health Vision for Action Evidence for Strategic Approaches. 2015;(January).

10. District B. UGANDA BUREAU OF STATISTICS INTERNATIONAL WOMENS , DAY CELEBRATIONS. 2019.

11. Survey H. Uganda. 2016.

12. Kawungezi PC, Akiibua D, Aleni C, Chitayi M, Kazibwe A, Sunya E, et al. MultiCenter Study in Upcountry Areas of Uganda. 2015;5(3):132-42.

13. Ewnetu W, Assegid S, Wondafrash B, Ewnetu H. Factors associated with late antenatal care initiation in an Ethiopian clinic. 2015;2.

14. Kisuule I, Kaye DK, Najjuka F, Ssematimba SK, Arinda A, Nakitende G, et al. Timing 
cognizancejournal.com

Kizito Omona et al, Cognizance Journal of Multidisciplinary Studies, Vol.1, Issue.3, March 2021, pg. 30-44

and reasons for coming late for the first antenatal care visit by pregnant women at Mulago hospital , Kampala Uganda. 2013;1-7.

15. Ranjit Kumar Research Methodology: A Step-by-step Guide for Beginners.2011.

16. Gebremeskel F, Dibaba Y, Admassu B. Timing of First Antenatal Care Attendance and Associated Factors among Pregnant Women in Arba Minch Town and Arba Minch District, Gamo Gofa Zone , South Ethiopia. 2015;2015.

17. Hanna G, Gulema H, Berhane Y. Timing of First Antenatal Care Visit and its Associated Factors among Pregnant Women Attending Public Health Facilities in Addis.

18. Bbaale E, Bbaale E. Factors influencing timing and frequency of antenatal care in Uganda. 2011;4:431-8.

19. Id TT, Chojenta C, Smith R, Loxton D. Factors affecting utilization of antenatal care in Ethiopia: A systematic review and meta- analysis. 2019;1-24. Available from: http://dx.doi.org/10.1371/journal.pone.0214848.

20. Gross K, Alba S, Glass TR, Schellenberg JA, Obrist B. Timing of antenatal care for adolescent and adult pregnant women in south-eastern Tanzania. 2012.

21. Okunlola MA, Owonikoko KM, Fawole AO, Adekunle AO. Gestational age at antenatal booking and delivery outcome. Afr J Med Med Sci [Internet]. 2008 Jun;37(2):165-169. Available from: http://europepmc.org/abstract/MED/18939401. 